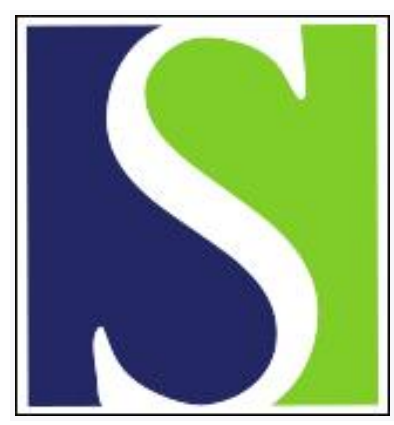

Scand J Work Environ Health 2007;33(5):321-323

https://doi.org/10.5271/sjweh.1152

Issue date: 31 Oct 2007

Does shift work cause cancer?

by Pukkala E, Härmä M

Affiliation: Finnish Cancer Registry, Institute for Statistical and Epidemiological Cancer Research, Liisankatu 21 B, FI-00170 Helsinki, Finland. [eero.pukkala@cancer.fi]

The following articles refer to this text: 2008;34(1):1-3;

2009;35(1):74-79

Key terms: cancer; editorial; shift work

This article in PubMed: www.ncbi.nlm.nih.gov/pubmed/17973057 


\section{Does shift work cause cancer?}

It is well-known that several work-related factors, for example, asbestos, benzene, nickel, wood dust or passive smoking in restaurants, can cause cancer. In Europe, 3.2 million new cases and 1.7 million deaths from cancer were reported in 2006 (1). Breast cancer has become the most common cancer diagnosed overall, with 430000 new cases in 2006 (13.5\% of all cancer cases). Traditional work-related risk factors are estimated to cause about $4 \%$ of malignant neoplasms among Nordic men but less than $1 \%$ among Nordic women (2).

While knowledge on the traditional risk factors for cancer has increased and many of these factors have successfully been eliminated from modern workplaces in developed countries, new environmental and individual risk factors for cancer have stepped forward. Shift work and work stress, the prevalent exposures of the "24-hour society", are among some of the "new" exposures now being intensively researched. The International Agency for Research on Cancer (IARC) is currently working on the evaluation of shift work as a possible carcinogenic risk to humans. The importance of "new" individual risk factors for cancer, alcohol intake, obesity, and the lack of physical activity that are sometimes linked to an occupational behavior model have also been acknowledged. The potential significance of shift work, work stress, and unhealthy living habits are not related to their high carcinogenic nature but, instead, to their extremely high prevalence. For example, if physical inactivity were estimated to increase the risk of breast cancer by $10 \%(3)$, about $3 \%$ of all cancers among women in Finland would be due to physical inactivity-a higher number of cases than those caused by all of the traditional occupational cancer risk factors combined.

Does shift work cause cancer? Before it can be decided that an environmental or occupational factor is carcinogenic, reliable and unbiased human evidence is needed to show a dose-response relationship between the studied exposure and the specific disease. In addition, it would make the interpretation easier if there were facts about a specific mechanism explaining the possible epidemiologic association.

The pineal gland is known to be the key organ in the regulation of the circadian clock. The suprachiasmatic nuclei of the hypothalamus, which are the major biological clocks in humans, regulate the excretion of plasma melatonin from the pineal gland. The information on external light is carried to the suprachiasmatic nuclei from the retina by the retinohypothalamic tract. Among people with normal circadian rhythmicity, the excretion of endogenous melatonin takes place only during the night, and the nighttime secretion can also be inhibited by artificial light. Melatonin is an important hormone in the regulation of circadian rhythms, but it seems to be involved also directly in the regulation of gonadal function (4). Decreased concentrations of circulating melatonin can result in an increased release of gonadotropins from the pituitary, stimulating testicular testosterone or ovarian estrogen production and release. Extensive animal data show that both pharmacological and physiological doses of melatonin can reduce the growth of malignant cells or tumors of the breast, prostate, and other tumor sites (5-7). On the other hand, light exposure during an experimental "night" of test animals increases the progression of cancer (8). In humans, breast cancer risk among females also decreases according to the degree of visual impairment ( 9 , $10)$; this decrease suggests a potential role and dose-response relationship between visual stimuli and the incidence of breast cancer. A similar, but less consistent trend has also been observed for prostate cancer in visually impaired males (9).

Although melatonin and light are indeed related to cancer, the relevance of the "melatonin theory" in the association between shift work and cancer depends on whether shift workers are exposed to 
decreased melatonin secretion due to changes in the environmental light-dark cycle. Shift work does induce changes in light exposure since night work has to be done under artificial lighting conditions and night workers must sleep and spend their free-time during the day. However, there seems to be no sufficient quantitative data on the actual 24-hour differences in light exposure between night and day workers. It is also known that night work changes the endogenous circadian rhythm of melatonin. Most studies indicate a partial phase delay in the melatonin rhythm during consecutive night shifts $(10,11)$ and a decrease in the melatonin circadian amplitude during the circadian adjustment process. In a recent study, night workers indeed showed a lower diurnal melatonin secretion than day workers (12).

This issue of the Scandinavian Journal of Work, Environment \& Health includes an epidemiologic study that suggests no effect of shift work on cancer risk (13). This study used registry data that basically included the entire Swedish working population. Jobs in the 1970 census were classified according to the estimated percentage of shift workers. The population was followed from 1971 to 1989 , for up to 19 years. The Swedish findings are not consistent with those of several earlier studies indicating an increased risk of prostate cancer among rotating shift workers and an increased risk of breast and colorectal cancer among female shift workers. For example, based on a systematic review and meta-analysis of 13 studies on night work and breast cancer risk between 1960 and 2005 (14), the aggregate relative risk estimate for all of the studies combined was 1.48, and, for female night workers not including those in studies of cabin crews, it was 1.51 .

The current study of Schwartzbaum et al (13) is based on a huge cohort of the Swedish working population. However, only a very small proportion of the population included shift workers, and therefore the power of the study is limited. As the authors state, a possible source of failure to observe a possible association between shift work and breast cancer may have been due to a misclassification of exposure as a result of the used aggregated, rather than individual, exposure information. Exposure was thus based on a group estimate made from job titles. Many of the workers classified as exposed may not have done night work at all, and some of those classified as unexposed may have worked at night. In the main analyses, job categories with $>40 \%$ shift workers ("exposed") were compared with job categories with $<30 \%$ shift workers ("unexposed"). The small exposure contrast may have further reduced the possibility of identifying a risk. Due to the increased diversity of workhours, night work is common in many occupations not recognized as actual night or shift work even by workers themselves. Furthermore, the maximum follow-up time of $<20$ years can be regarded as short since all studies on shift work and breast cancer with a longer follow-up time ( $\geq 20$ years) have shown an increased risk (15-17).

Schwartzbaum et al (13) were not able to control for potential confounding factors other than marital and socioeconomic status. For instance, in breast cancer data, the most important cofactors, age at first delivery, number of children, and use of hormone replacement therapy, were not yet available in the old combined data set used in this study. Of the other risk factors of breast cancer, obesity and smoking, are normally more frequent among shift workers than among day workers (18), and the lack of control of these two factors probably would not have changed the null result.

In general, there are even opinions that low relative risks should not be studied at all epidemiologically, one of the reasons being the possibility that even a modest uncontrolled residual confounding may falsify the conclusion. On the other hand, some of the factors with a potential low excess of adverse health effects are so common that even a very small relative excess may have larger public health effects than the high relative risks related to rare exposures. Studies on low relative risks are a challenge to epidemiologists, but maybe not impossibly so, especially in the Nordic countries when additional selection of register-based cofactor data and virtually complete follow-up procedures of exposed persons are possible.

In conclusion, Schwartzbaum et al (13) bring another, even rather unfitting piece to the large puzzle trying to shape the relationship between shift work and cancer, and it is important that this puzzle piece be published, even with the shortcomings described by the authors themselves and others put forth in 
this editorial. The collective picture of the earlier studies on shift work and cancer is too consistent to be dismissed by a single ecological study supporting the null hypothesis. A special challenge is issued to the ongoing, more advanced Nordic studies that have a basic setting similar to that of the study by Schwartzbaum et al (13), but will have a follow-up of up to 45 years, markedly more cancer cases from all five Nordic countries, better classification of job exposure, and data on the most important cofactors. Additional data are also needed on the effect of shift work systems on light exposure and melatonin secretion.

\section{References}

1. International Agency for Research on Cancer (IARC). New European cancer figures-World Cancer Agency says major efforts needed toward prevention in Europe. Geneva: World Health Organization; 2007. Press release no 174, 7th February 2007.

2. Olsen J, Andersen A, Dreyer L, Pukkala E, Tryggvadottir L, Gerhardsson de Verdier M, et al. Summary of avoidable cancers in the Nordic countries. In: Olsen J, Andersen A, Dreyer L, Pukkala E, Tryggvadottir L, Gerhardsson de Verdier M, et al, editors. Avoidable cancers in the Nordic countries. APMIS 1997;105 suppl 76:141-6.

3. Rintala PE, Pukkala E, Paakkulainen HT, Vihko VJ. Self-experienced physical workload and risk of breast cancer. Scand J Work Environ Health. 2002;28(3):158-62.

4. Davis S, Mirick D. Circadian disruption, shift work and the risk of cancer: a summary of the evidence and studies in Seattle. Cancer Causes Control. 2006;17:539-45.

5. Cos S, Mediavilla MD, Fernandez R, Gonzalez-Lamuno D, Sanchez-Barcelo EJ. Does melatonin induce apoptosis in MCF-7 human breast cancer cell in vitro? J Pineal Res. 2002;32:90-8.

6. Siu WE, Lau KW, Tam PC, Shiu SY. Melatonin and prostate cancer cell proliferation: interplay with castration, epidermal growth factor, and androgen sensitivity. Prostate. 2002;52:106-22.

7. Kanishi Y, Kohayashi Y, Noda S, Ishizuka B, Saito K. Differential growth inhibatory effect of melatonin on two endometrial cancer cell lines. J Pineal Res. 2000;28:227-33.

8. Pukkala E, Ojamo M, Rudanko S-L, Stevens RG, Verkasalo PK. Does incidence of breast cancer and prostate cancer decrease with increasing degree of visual impairment. Cancer Causes Control. 2006;17:573-6.

9. Verkasalo P, Pukkala E, Stevens R, Ojamo M, Rudanko S. Inverse association between breast cancer incidence and degree of visual impairment in Finland. Br J Cancer. 1999;80:1459-60.

10. Koller M, Härmä M, Laitinen J, Kundi M, Piegler B, Manfred H. Different patterns of light exposure in relation to melatonin and cortisol rhythms and sleep of night workers. J Pineal Res. 1994;16:127-35.

11. Härmä M. Circadian adaptation to shift work: a review. In: Hornberger S, Knauth P, Costa G, Folkard S, editors. Shiftwork in the 21st century: challenges for research and practice. Frankfurt/M, Berlin, Bern, Bryssel, New York, Oxford, Wien: Peter Lang; 2000. p 125-30. Arbeitswissenschaft in der betrieblichen Praxis, vol 17.

12. Hansen AM, Grade AH, Hansen J. Diurnal urinary 6-sulfatozymelatonin levels among healthy Danish nurses during work and leisure time. Chronobiol Int. 2006;23:1203-15.

13. Schwartzbaum J, Ahlbom A, Feychting M. Cohort study of cancer risk among male and female shift workers. Scand J Work Environ Health. 2007;33(5):336-43.

14. Megdal SP, Kroenke CH, Laden F, Pukkala E, Schernhammer E. Night work and breast cancer risk: a systematic review and meta-analysis. Eur J Cancer. 2005;41:2023-32.

15. Lie JA, Roessing J, Kjaerheim K. Breast cancer and night work among Norwegian nurses. Cancer Causes Control. 2006;17:39-44.

16. Schernhammer ES, Kroenke CH, Laden F, Hankinson SE. Night work and risk of breast cancer. Epidemiology. 2006;17:108-11.

17. Schernhammer ES, Laden F, Speizer FE, Willet WC, Hunter DJ, Kawachi I, Golditz GA. Rotating night shifts and risk of breast cancer in women participating in the nurses' health study. J Natl Cancer Inst. 2001;93:1563-8.

18. Härmä M. Workhours in relation to work stress, recovery and health [review]. Scand J Work Environ Health. 2006;32(6):502-14.

Eero Pukkala, PhD

Finnish Cancer Registry

Institute for Statistical and Epidemiological

Cancer Research, Finland
Mikko Härmä, MD

Centre of Expertise on Human Factors at Work Finnish Institute of Occupational Health

Helsinki, Finland 
